\title{
A mission management system for a fleet of gliders
}

\author{
Magali BARBIER, \\ Eric BENSANA, \\ David DOOSE \\ ONERA/DTIS \\ Toulouse, FRANCE \\ Firstname.Surname@onera.fr
}

\author{
Florent BESSON, \\ Yann LE PAGE \\ ALSEAMAR \\ Rousset, FRANCE \\ InitialfirstnameSurname@alseamar- \\ alcen.com
}

\author{
Mikaël LEOPOLDOFF \\ CENTRAL WEB \\ La Valette Du Var, FRANCE \\ MLeopoldoff@centralweb.fr
}

\begin{abstract}
The objective of AGLIMMS project, whose acronym stands for Acoustic GLIders Mission Management System, is to efficiently coordinate a fleet of underwater gliders whose missions are to obtain physical, chemical, biological and/or acoustic measurements on a large 3D sea area. This paper describes planning and supervision functions under development and their integration in a global centralised architecture. A demonstration with three SeaExplorer from Alseamar is planned late 2019.
\end{abstract}

Keywords-underwater glider, fleet, mission management system, planning, supervision

\section{INTRODUCTION}

Oceanographic missions performed by underwater gliders are various [1]: climate change monitoring, analysis of human activity (oil and mining industries), study of local flora and fauna, seabed mapping for its exploitation and protection..

Whereas missions assigned to most Autonomous Underwater Vehicles last several days on several square dozen kilometres, underwater gliders can stay at sea for several months and scan thousands of kilometres. These lowcost vehicles move silently while interfering very little with their environment and their low weight allows light deployments and recoveries. Their drawbacks are a bad underwater positional accuracy in hundreds of meters and their limited power capacity, a few watts used for travelling (by changing buoyancy and using winged surfaces to produce a sawtooth-like trajectory) and for payload. When in mission, they surface every few hours for measurement data transmission and potential trajectory update. They are controlled by an experienced pilot who is alerted each time a glider surfaces and who can adapt the trajectory or change the mission if needed.

Using a fleet (sometimes called a network) of gliders either increases the spatial acquisition or reduces the processing time. The probability to detect sources of signals (gas leak, marine mammals, anthropogenic noise...) increases and positional accuracy is better thanks to a possible triangulation. Specific patterns such as comb allow for a better coverage of spatial areas as all synchronised gliders evolved in the same environment. Lastly, fleet missions can potentially proceed when a glider leaves the team, especially when reconfiguration issues are managed.

For an efficient coordination, the management of a fleet of gliders however requires more than individual experienced pilots. Many types of heterogeneous data must be assimilated: they stem from platforms, from the run of models (e.g. currents, sound propagation) and from external world (e.g. automatic ship identification systems AIS, bathymetry, weather). A centralised global Mission Management System (MMS) is thus required to help the pilots, by leaving them only high level tasks while algorithms perform the coordination activities.

Four types of underwater gliders are on the market: SeaExplorer from Alseamar, Seaglider from University of Washington, Spray from Scripps Institution of Oceanography, and Slocum from Teledyne Webb Research. Several fleet experiments [2] [3] [4] [5] have been achieved with a fleet of above gliders, which were either individually operated by pilots discussing together or virtually moored.

The structure of the paper is as follows. Section II presents AGLIMMS project in which this work falls. Section III introduces the Planning and Supervision System (PSS) and an illustrative example, then section IV the glider action cost model. Sections V and VI explains respectively planning and supervision problems then proposed solution algorithms. Section VII shows current PSS implementation in MMS before highlighting future work in section VIII.

\section{AGLIMMS PROJECT}

Three complementary partners are involved in the French AGLIMMS project (2016-2019): Alseamar (project lead) operate SeaExplorer (Fig. 1) since 2017; ONERA do extensive research on unmanned vehicles cooperation since 2007; CentralWeb develop big data management and webmapping systems.
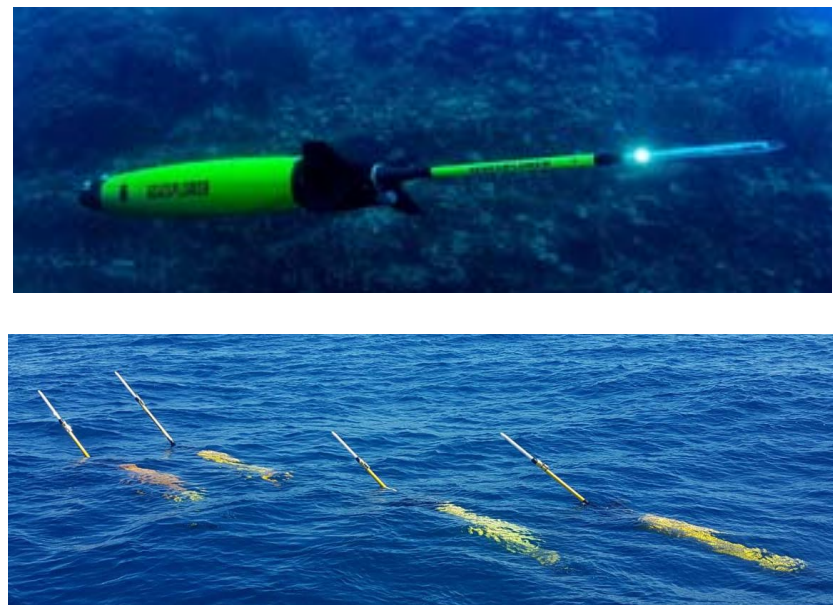

Fig. 1. SeaExplorer underwater gliders from Alseamar. 
The objective of AGLIMMS is to build a centralised MMS to efficiently coordinate a fleet of gliders which needs are:

- reduced work for pilots who thus have rather a role of mission operators: need of a Man Machine Interface (MMI) allowing the definition of high level objectives and development of algorithms to compute a plan for the fleet of gliders and to supervise their sea execution;

- sea performance: optimised planning software for gliders coordination, online supervision of sea execution and online plan adaptation when surfacing;

- integration in the real world: use of environmental data and models, navigation models, consideration of data coming from real gliders via satellite links...

- Technology Readiness Level between 4 and 5: a demonstration with three SeaExplorer is planned late 2019.

This paper focuses on the planning and supervision functions of MMS.

\section{PlanNING AND SUPERVISION SySTEM (PSS)}

AGLIMMS PSS aims at managing the coordination of all gliders actions for the achievement of missions in an optimised way. Fig. 2 shows interactions between planning and supervision functions and within the MMS.

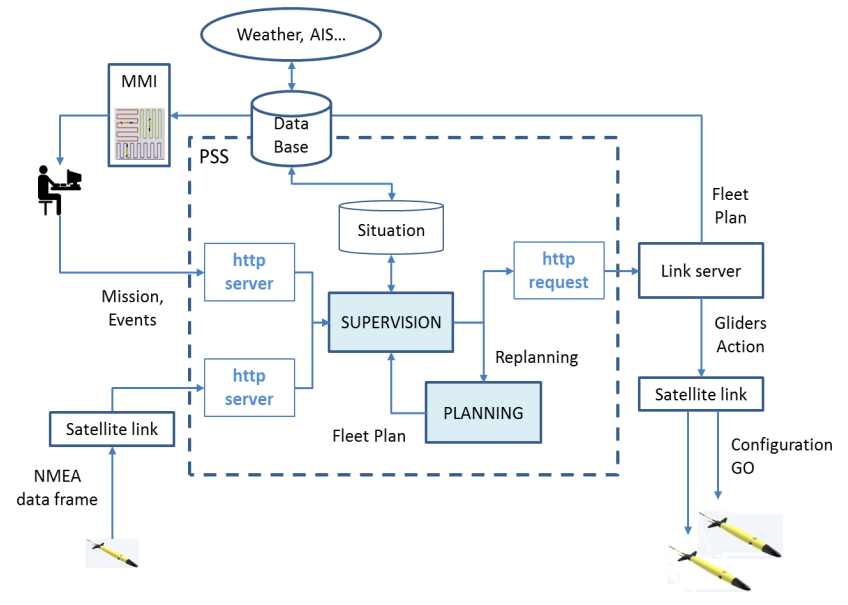

Fig. 2. Planning and Supervision System.

A fleet mission defined by an operator contains: the navigation area, an initial set of gliders (gliders can enter and leave an ongoing mission), several phases, and constraints between these phases. Five types of phases are available amongst them path following (a path being defined by a set of waypoints), 4D systematic coverage for data gathering, and $4 \mathrm{D}$ random coverage for signal detection.

Fig. 3 shows an ongoing mission example that is used thereafter to illustrate the PSS functions. Glider $\mathrm{g} 1$ follows a path whereas gliders g2 and g3 are performing an acoustic barrier in midwater; g1 is underwater and its position is estimated, g2 and g3 are navigating around the mooring point and their position adequately known. Next phases are systematic coverages of two areas, one with two gliders in a comb formation amongst them $\mathrm{g} 1$, and one with one glider. In this example, the assignment of $\mathrm{g} 1$ to one coverage phase is imposed by the operator whereas g2 and g3 assignments to the coverage phases are not definitive: they can change each time a plan is computed (this matter is symbolized by a question mark on figures). Final step for all gliders are to join a recovery area then performing another acoustic barrier. Fig. 4 shows the related graph of phases.

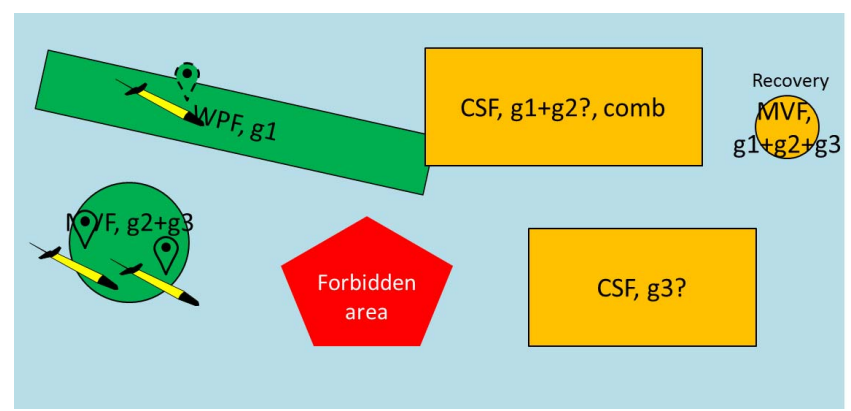

Fig. 3. Example of current state of a fleet mission: areas of running phases are in green, pending in orange. Gliders estimated positions are in solid line, estimated in dashed line.

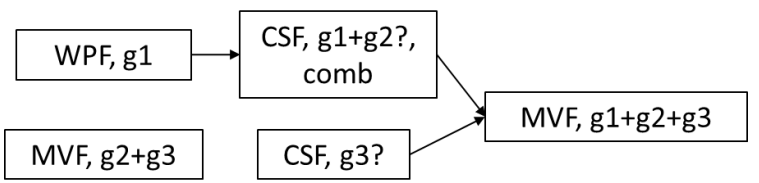

Fig. 4. Phases of the example mission. WPF = path following, MVF = virtual mooring, $\mathrm{CSF}=$ systematic coverage. Gliders are g1, g2 and g3.

\section{GLIDER ACTION COST MODEL}

Optimised computation of plans requires the knowledge of the cost of one glider action when moving in its environment. Input data are the hydrodynamic model (reversed from real data), the maximal size of ballasts, their centring, electrical consumption model of the glider and of its payloads when used. Planning criteria relate to duration, time interval, consumption, and distance. Environment data are sea currents maps, bathymetry maps and potential forbidden areas.

A "glider action" (also further called "surfacing action") is composed of a sawtooth-like navigation profile between two surfacing points whose main variables are: vertical speed $\mathrm{Vz}$, slope $\gamma$, bottom immersion $\mathrm{Zb}$, top immersion Zt (as a glider does not surface after each ascent) and number of descent-ascent patterns Sr. Above side of Fig. 5 shows this profile without currents. As sea gliders are sensitive to currents that vary according to depth, the navigation profile may adapt to current layers; example on below side of Fig. 5 shows a $2 \mathrm{D}$ display but trajectories are $4 \mathrm{D}$ with $\mathrm{X}, \mathrm{Y}, \mathrm{Z}$ and time. 

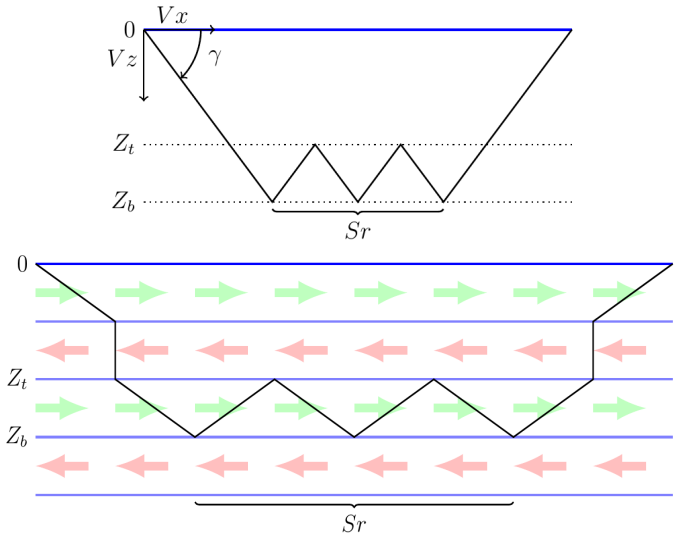

Fig. 5. Navigation profile without (above) and with (below) currents (2D display).

In what follows, we distinguish a "surfacing action" (glider action given by its navigation profile as previously defined) from the "surfacing waypoint" (only its final position).

\section{PlanNING FUnCTION}

The planning function computes a "fleet plan" composed of all individual glider plans. A "glider plan" is one sequence of all glider actions from now up to end of mission or to a specific date. Planning algorithm can be called whenever the current situation evolves (see section VI on supervision), which generally leads to a regular update of the fleet plan.

Current fleet plan is used to make gliders dive. Each new fleet plan is displayed on the MMI. Offline, this displayed plan allows an operator to iteratively prepare new fleet missions; online, it allows them to potentially react to current situation.

Need for surfacing comes firstly from mission (surfacing needed for every heading changes in path), area coverage, specific operator constraints...) and secondly from navigation performing. The planning function uses then two algorithms, A1 calling A2 when necessary:

- $\mathrm{A} 1$ = fleet mission planning: selection of phases to perform, assignment of gliders to phases, choice of the sequence of phases visited by each glider, choice of the pattern and splitting for each phase and the computation of surfacing waypoints to answer mission constraints (further called "mission surfacing waypoint");

- $\quad$ A2 = glider navigation planning: computation of all surfacing actions to follow above mission surfacing waypoints while considering gliders characteristics and environmental constraints. This algorithm most of the time introduces new waypoints called "navigation surfacing waypoints". Note that mission surfacing waypoints are also navigation ones because the navigation profile has also to be computed from and towards them.

These two algorithms are illustrated for the example described in section III. Intermediate (because it does not represent a real plan) result of one fleet mission planning A1 is shown on Fig. 6 whereas result of several navigation planning A2 between each couple of mission surfacing waypoints is shown on Fig. 7. The final fleet plan includes all the surfacing actions.

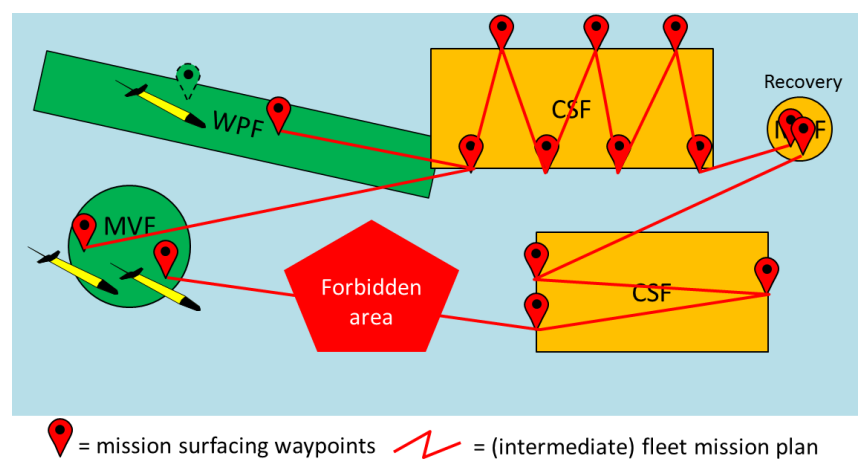

Fig. 6. Fleet mission planning A1: mission surfacing waypoints and the computed plan are in red.

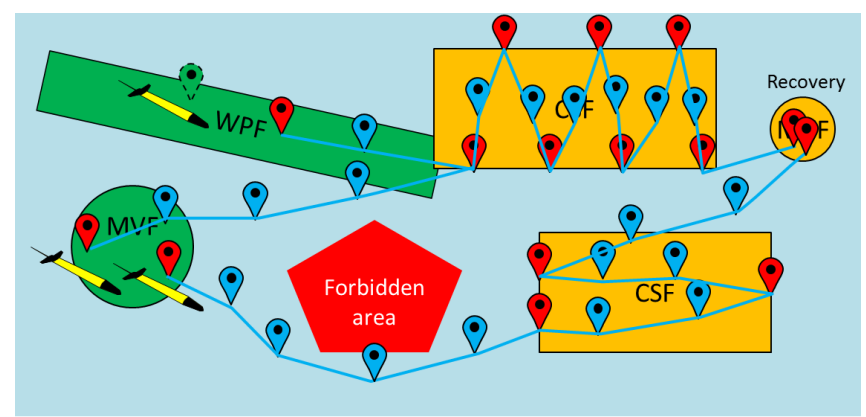

$\vartheta$ = navigation surfacing waypoints

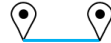

glider action (navigation profile)

Fig. 7. Glider navigation planning A2: navigation surfacing waypoints and computed plans (between couples of mission surfacing waypoints) are in blue.

Current hypothesis for the definition of the fleet mission planning problem are: different types of gliders, temporal constraints between phases, phases that can be defined as optional, number of gliders needed for each phase or specific gliders already assigned to phases by the operator, wanted formation or not when more than one glider (if a formation is imposed we assume that the set of gliders is given), temporal information about starting and finishing times of each phase.

The fleet mission planning problem (A1) is modelled from the current mission definition and the current situation of the fleet as a Constraint Satisfaction Problem (CSP):

- The current mission definition (coming from the operator through the MMI) defines the current set of phases to be performed on a given horizon as well as logical temporal constraints between phases. This definition may also contain constraints on some aspects of the problem definition (intervals of values for start date as well as end date, phases pattern can be free or not, intermediate transit can be implicit or not...)

- The current fleet situation built from data collected from gliders and ongoing mission execution gather all information about the current status of each glider: true position if on surface or expected position and time of next surfacing, estimated status of battery level, current phase... 
The global model embeds several sub-parts dedicated to specific aspects of the problem:

- Flow variables and constraints for gliders (as each glider visits a subset of the set of phases) and phases route definition;

- Assignment variables and constraints to select the set of gliders that will be used to perform each phase;

- Temporal variables and constraints bearing on starting time, duration and ending time for phases;

- Temporal and spatial constraints for each glider path definition (sequence of mission surfacing waypoints) taking into account pattern, formation and splitting aspects;

- Consumption variables and constraints for each glider involved in the mission.

These sub-problems are connected through the set on decision variables (mainly Booleans) defining the possible assignment of each glider to a phase and the possible flow of gliders between couples of phases.

Satisfaction algorithms can be used to solve this CSP. Moreover by defining a cost function based on some variables of the CSP, optimization can be performed too. The chosen framework for building and solving the CSP is the $\mathrm{CHOCO}$ tool [6]. In a second phase we intend to test the possible use of the inCELL framework [7].

Inputs for the glider navigation planning problem A2 are: glider data, initial and final mission surfacing waypoints, glider action cost model introduced in section IV, 3D currents data (direction and speed by depth layers), bathymetry data (depth), and forbidden areas. The environment is meshed in cells. Output is the sequence of navigation surfacing waypoints between the two given mission surfacing waypoints.

Three algorithms have been implemented to solve A2. The first one gives quickly a solution then slowly the optimal one by browsing the tree structure of solutions to optimise each next waypoint looking for the global plan cost. It could be used offline during the preparation of a fleet mission. The second is a kind of $\mathrm{A}^{*}$ algorithm with a traversal study of the tree structure of solutions; a first solution is obtained slowly but the optimal one quickly. It could be used when all goes well during mission. The third, a Monte Carlo Tree Search (MCTS), is efficient for the first waypoint but next waypoints have to be forced to obtain a global plan. It could be used when real and estimated trajectories are significantly different which means a quick next action is required.

An example of a result of the fist algorithm is shown on Fig. 8. These algorithms will be evaluated on real data and within the fleet mission planning A1.

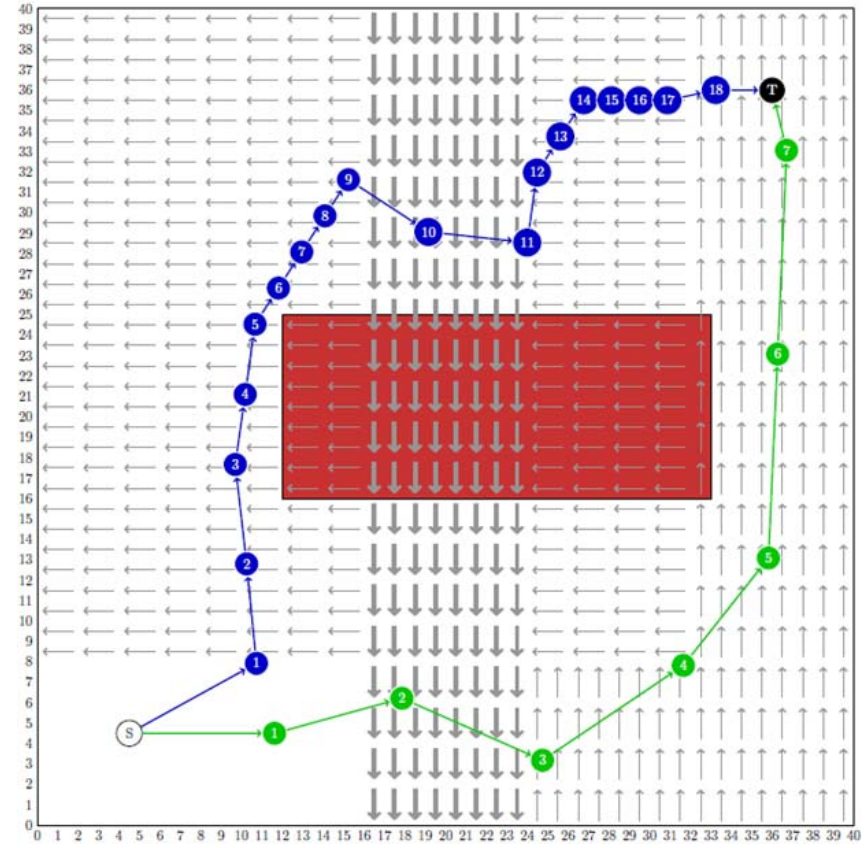

Forbidden area

$\rightarrow$ Currents (intensity increases with width)

- Initial mission surfacing waypoint of the glider

- Final mission surfacing waypoint of the glider

- Navigation surfacing waypoints: quick initial trajectory

- Navigation surfacing waypoints: final optimised trajectory

Fig. 8. Cartesian XY view of result of a glider navigation planning (A2) between two mission surfacing waypoints. Resolution is 3D.

\section{SUPERVISION FUNCTION}

The supervision function triggers planned gliders actions at computed diving times and follows their execution. When a glider surfaces, it checks the current situation and selects next appropriate steps: triggering a glider dive, updating glider navigation plan, updating fleet mission plan. The supervision function can then call the planning function (see section $\mathrm{V}$ ) to update/enhance a plan.

More broadly, asynchronous events received by supervision and which could trigger this replanning process (cf. Fig. 2) come from gliders but also from the operator and from the environment (via the database). Events can concern one or several gliders and one or several phases. For a given event, different strategies can be applied. Three types of events are identified:

- Extreme events leading to mission stop: stop order from the operator, end mission in a near future, and no planning solution found. This type of event turns off the supervision of all other events.

- Events which reaction concerns the fleet: leaving order to gliders from the operator (whatever the reason), entering order of new gliders from the operator, graph of phases modified by the operator, maximal duration reached for one phase, acoustic detection, new environment data available, and critical problem of a glider when surfacing. This type of events systematically leads to the triggering of a fleet mission planning. 
- Events which reaction could concern only one glider: surfacing of a glider, availability of a glider for diving (after data loading), and anticipation of glider dive for safety reason. This type of events could trigger a fleet mission planning or lead to no reaction.

To react correctly to these asynchronous events, the supervision function must monitor mission situation:

- Executed, disrupted, or discarded phases are removed from the graph of phases.

- List of gliders contains those that the PSS can supervise.

- Current fleet plan is the last one computed by the planning function.

- Main glider state includes last diving data, next navigation waypoint and relative action (when under sea), next diving time (when in surface), ongoing phase, battery data.

- Main phase state includes ongoing or pending status, start date (real or planned), assigned gliders, and area (remaining area to be covered for coverage phases).

These situations, their updates and their reactions are managed through the formalism of Petri nets that allows the composition of elementary behaviours. A Petri net [9] is a bipartite graph with two types of nodes: places model the state of the system and transitions model its evolution. Petri net PSS loop on Fig. 9 shows the call of planning function when an event occurs. Current state of this Petri net is given by a black token in the PSS_WAIT place. When an event occurs (triggering of event2assess transition), the current situation is assessed (token moves from PSS_WAIT place to SIT_ASSESS place); a decision is taken by supervision either to do nothing (assess2wait transition puts the net back in the PSS_WAIT state) or to call the planning function (assess $2 \mathrm{pln}$ transition from SIT_ASSESS place to MISS_PLN place); when correctly computed (PLNok2react transition), the planned reaction is ran (place REACTION, behaviour is in other Petri nets).

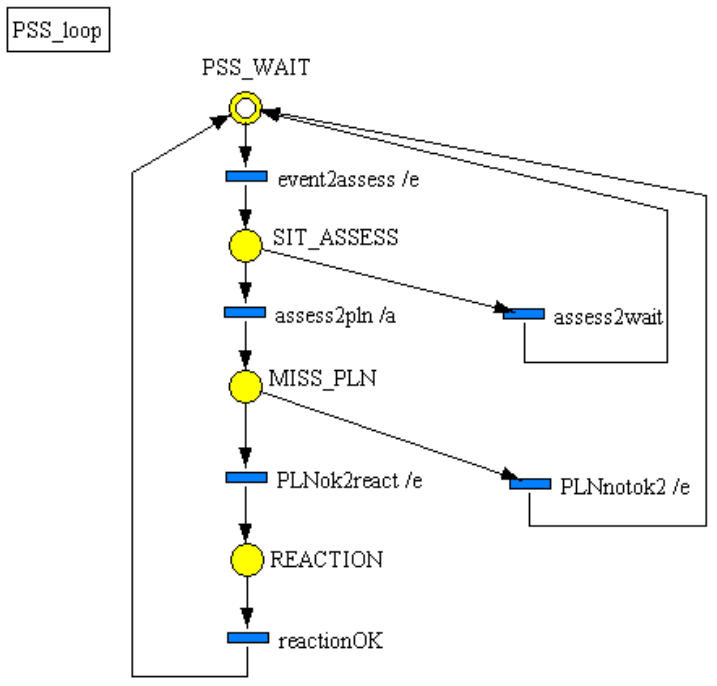

Fig. 9. Example of Petri net modelling in the supervision function.

\section{IMPLEMENTATION WITHIN MMS}

MMS is centralised on a web-based platform which allows multiple users to prepare, supervise and analyse gliders missions. This web-based platform is described in [10]. Its actual version has been successfully tested in real conditions for the human piloting of gliders. Next step is to connect the PSS.

As supervision function is central in the PSS architecture, it thus carries out connections within MMS (see Fig. 2). To simulate the occurrence of events and evaluate PSS architecture, a client-server application has been developed. The server is the PSS that supervises the mission according to received events. The client is a especially created web page that allows sending asynchronous events towards the PSS server.

Running this simulation test-bed will complete standalone tests of the planning and supervision functions.

\section{CONCLUSIONS AND FUTURE WORK}

AGLIMMS project is in its final year. Planning and supervision functions have been specified, with parameters, constraints, variables and criteria for the first, and event and behavioural reactions for the second. Planning algorithms and supervision mechanisms are under development in parallel with standalone tests. Connection within the MMS is simulated through a client-server application.

Work is the next months prior to demonstration will be focused on the validation of the Planning and Supervision functions based on realistic test cases.

A demonstration with three SeaExplorer operating in a formation is planned late 2019 in the Mediterranean Sea. It will highlight the whole communication process, the PSS reaction to real events, and the ground replanning process to update the fleet mission plan. The scenario will be close to the example given in this paper with path following and systematic coverage phases.

Further work on this thematic could be to integrate the fleet of gliders in a global system that will include vessels and other types of autonomous vehicles and require for instance global planning capacities, to consider heterogeneous gliders and various payloads, to implement more generic team strategies and in relation to enhance communication strategies, to mathematically prove system robustness, and last but not least to onboard implement some decisional autonomy.

\section{ACKNOWLEDGEMENT}

This work is partially founded by the French defence procurement agency DGA.

\section{REFERENCES}

[1] Rudnick, DL, 2016: Ocean research enabled by underwater gliders. Annu. Rev. Mar. Sci., 8, 519-541, doi:10.1146/annurev-marine122414-033913.

[2] Fratantoni, DM, 2005: Development of oceanographic sampling networks using autonomous gliding vehicles and demonstration of WHOI glider fleet operations in the tropical western Pacific for the Naval Oceanographic Office. Research gate.

[3] Leonard, NE, Paley, DA, Davis, RE, Fratantoni, DM, Lekien, F, Zhang, F, 2010: Coordinated control of an underwater glider fleet in 
BARBIER, ONERA, A mission management system for a fleet of gliders

an adaptive Ocean sampling field experiment in Monterey Bay. Journal of Field Robotics 27(6), 718-740, doi: 10.1002/rob.20366.

[4] Real-Arce, DA, Quevedo, E, Barrera, C, Hernandez, J, Llinas, O, Barbero, F, Morais, A, 2016: A new integrated border security approach: the FP7 PERSEUS project. Marine Technology Society Journal, Volume 50, Number 4, July/August, pp. 14-25(12), doi: $\underline{10.4031 / \mathrm{MTSJ} .50 .4 .12}$

[5] Onken, R, Fiekas, HV, Beguery, L, Borrione, I, Funk, A, Hemming, $\mathrm{M}$, et al., 2018: High-resolution observations in the western Mediterranean Sea: the REP14-MED experiment. Ocean Sci., 14, 321-335, doi:10.5194/os-14-321-2018.

[6] Prud'Homme, C, Fages, JG, and Lorca, X, 2017: Choco Documentation, TASC-LS2N CNRS UMR 6241, Costling S.A.S., http://www.choco-solver.org.
[7] Pralet, C and Verfaillie, G, 2013: Dynamic online planning and scheduling using a static invariant-based evaluation model. ICAPS. aaai.org.

[8] Lesire, C and Pommereau, F, 2018: ASPiC: an acting system based on skill Petri net composition. IROS, doi:10.1109/IROS.2018.8594328.

[9] Murata, T. (1989). Petri nets: properties, analysis and applications. IEEE. 77(4), 541-580, doi:10.1109/5.24143.

[10] Besson, F, Pasqueron, O, Romero, J, Barbier, M, Bensana, E, Doose, D, Leopoldoff, M, and Larrasoain, S, et al, 2019: A centralized webbased platform for combined glider and satellite observation analysis. OCEANS 2019, in press. 